\title{
Concurrent Partnerships and HIV Risk among Men Who Have Sex with Men in New York City
}

\author{
Hong Van Tieu, MD, MS, Vijay Nandi, MPH, Victoria Frye, DrPH, Kiwan Stewart, Heriberto \\ Oquendo, Blaz Bush, Magdalena Cerda, PhD, Donald R. Hoover, PhD, Danielle Ompad, \\ PhD, and Beryl A. Koblin, PhD on behalf of On behalf of the NYC M2M Study Team
}

\section{Abstract}

Background-Concurrent partnerships are a significant public health concern among men who have sex with men (MSM). This study describes the prevalence of concurrency and its association with serodiscordant/serostatus unknown unprotected anal or vaginal intercourse (SDUI) among MSM in New York City.

Methods-1,458 MSM completed a social and sexual network inventory about their male and female sex partners, including concurrency, in the last 3 months. Logistic regression identified factors associated with SDUI.

Results-Median age was 29 years. $23.5 \%$ reported being HIV+. The men reported mean 3.2 male partners in last 3 months. 16.6\% reported having recent SDUI. 63.2\% described having concurrent sex partners (individual concurrency based on overlapping dates of relationships); $71.5 \%$ reported having partners whom they believed had concurrent partners (perceived partner concurrency); $56.1 \%$ reported that both they and their partners had concurrent partners (reciprocal concurrency). Among HIV+ men by self-report, having SDUI was positively associated with individual concurrency, any alcohol use during sex, having more male sex partners, and not having a main partner. Among self-reported HIV- men, having SDUI was positively associated with perceived partner concurrency, lower education level, any alcohol and drug use during sex, having more male sex partners, and having an anonymous partner.

Conclusions-Concurrency was common among MSM. The association of SDUI with individual and perceived partner concurrency, along with substance use during sex, having an anonymous partner, and having many sex partners likely further increases HIV acquisition and transmission risk among MSM. HIV prevention interventions should address concurrency among MSM.

\section{INTRODUCTION}

Concurrent partnerships (in which sex with a partner occurs between two sexual intercourse acts with another partner) ${ }^{1-3}$ are of public health concern among men who have sex with men (MSM). Concurrency can accelerate HIV and other sexually transmitted infections

Correspondence: Hong Van Tieu, MD, MS, Laboratory of Infectious Disease Prevention, Lindsley F. Kimball Research Institute, New York Blood Center, 310 E. $67^{\text {th }}$ Street Suite 3-110, New York, NY 10065, Telephone 212-570-3081.

The authors do not report any conflicts of interest. 
(STIs) spread by expanding sexual network size, enhancing network connectivity, and increasing rate and efficiency of infectious disease transmission within and across networks before individuals have knowledge of or treatment for an infection. ${ }^{4-11}$ Concurrency was found in many, ${ }^{12-14}$ but not all, ${ }^{15-17}$ studies to be important in HIV and STI spread among heterosexuals.

Although multiple sex partners is a recognized risk for HIV infection among MSM, ${ }^{18}$ data on concurrency among MSM, particularly in the U.S., are limited. ${ }^{5,19}$ MSM who reported concurrency in San Francisco had more sexual encounters per partnership and unprotected sex than men who did not report concurrency. ${ }^{19}$ MSM in concurrent partnerships in Maryland were more likely to have undiagnosed HIV infection. ${ }^{21}$ Other studies of MSM had differing results on concurrency and STI associations. ${ }^{22,23}$

This study aims to describe the prevalence of concurrency among New York City (NYC) MSM and examine its association with unprotected serodiscordant intercourse.

\section{METHODS}

\section{Study Sample and Recruitment}

NYC M2M was a cross-sectional study to identify urban environment characteristics that influence sexual risk behaviors, substance use and depression among MSM in NYC. ${ }^{24} \mathrm{MSM}$ were recruited in person using a modified venue-based, time-space sampling methodology; internet- and mobile application-based recruitment was also used. ${ }^{25}$ In-person recruitment occurred at locations (e.g., street locations, retail businesses, bars and clubs) during sampling events. Men were systematically approached and screened for preliminary eligibility. Eligible participants were invited to provide contact information. For website and mobile application recruitment, interested participants were referred to the NYC M2M website and screened for preliminary eligibility; those eligible were asked to provide contact information. Attempts were made to contact preliminarily eligible participants to further screen for eligibility and schedule an on-site study visit.

Eligibility criteria included: biological male at birth, $\geq 18$ years old, NYC residents, engaged in anal sex with a man in the past 3 months, communicated in English or Spanish. The four institutional review boards of the co-investigators approved the study.

\section{Study visit}

After providing written informed consent, participants completed a questionnaire using audio computer-assisted self-interview (ACASI) technology (average 60-90 minutes). Next, a social and sexual network questionnaire (SSNQ) was completed with an interviewer with data entry into a computer system (average 35 minutes). Participants then received HIV risk reduction counseling and were offered a rapid HIV antibody test, with reactive HIV tests confirmed by Western Blot. Participants testing HIV-positive were referred for treatment and services. Participants were reimbursed \$50 and a Metrocard. 
Sociodemographics-Sociodemographics collected on the ACASI questionnaire included age, race/ethnicity, self-identification as Latino/Hispanic, education, household income, marital status, and incarceration history.

HIV and STI-The ACASI questionnaire included HIV testing history, self-reported HIV serostatus, and STI history (syphilis, genital or rectal gonorrhea or Chlamydia, new genital or rectal herpes infection in the last 3 months). Results from HIV testing at the study visit were designated as negative, positive, or refuse to test/unknown.

Risk Behaviors-Use of alcohol, injection drugs, and non-injection drugs in the last 3 months was collected on the ACASI questionnaire. Specific drug or alcohol use during protected and unprotected sex in the last 3 months with each partner was obtained from the $S S N Q$. Information about sexual partners and sexual risk behaviors were obtained from the SSNQ. Participants were first asked, using a name generator, to name up to 10 people with whom they have had a social relationship and up to 15 sexual partners with whom they have had anal or vaginal sex in the last 3 months, with an approximation of how many additional sex partners if the participant had $>15$ in the last 3 months. Questions were asked about each named sexual partner: (1) sociodemographics, gender, (2) HIV status, (3) partner type, (4) where participant met the partner, and (5) frequency of anal or vaginal sex and condom use with sex with the partner in the last 3 months. The partner's HIV status was based on the participant's belief about his partner's HIV serostatus.

Concurrency-Concurrency measures were derived from the SSNQ. Individual concurrency (participant reports that he is having another sex partner during a period that overlaps with his sexual relationship with a partner) was assessed by two methods. First, the participant was asked about each partner: "How many other people did you have anal or vaginal sex with while you were sexually involved with [name] in the past 3 months?" If the participant answered $>1$ partner, then he was classified as having individual concurrency based on the single question. In addition, the participant was asked about start and stop dates (in month and year) of the sexual relationship: "When was the first/last time you had anal or vaginal sex with [name]?" If the participant reported an overlap of at least 1 month among sex partners in response to these two questions, he was classified as having individual concurrency based on dates. Perceived partner concurrency (participant believes that his sex partner is having sex with another partner during the time that overlaps with his sexual relationship with that partner) was assessed from the participant's answer to the question: "Did you believe [name] had other sexual partners while you were sexually involved with [name] in the past 3 months?" Reciprocal concurrency was defined as having both individual and partner concurrency.

Outcome-Serodiscordant/serostatus unknown unprotected anal or vaginal intercourse (SDUI) was defined as having unprotected intercourse (anal and/or vaginal) with a male, female, or transgender sex partner in the last 3 months with HIV serodiscordance or serostatus unknown, and was dichotomized as any or no SDUI. The participant's HIV status was determined by self-reported HIV status on the ACASI questionnaire prior to HIV 
testing at the site. Intercourse was defined as unprotected if the participant reported not always using a condom during anal or vaginal sex with a partner in the past 3 months.

\section{Statistical Methods}

Sociodemographic and risk behavior characteristics, HIV serostatus, and prevalence of individual, perceived partner, and reciprocal concurrency were tabulated. A kappa coefficient was calculated to assess the beyond-chance agreement between the two measures of individual concurrency, with a pre-determination to utilize the individual concurrency measure based on dates if kappa was high. ${ }^{1}$ Correlation between individual and perceived partner concurrency was calculated using Phi coefficient. Differences in prevalence of individual, perceived partner, and reciprocal concurrency were compared by baseline sociodemographic characteristics and HIV serostatus using Chi-square or Fisher's exact tests. Bivariable logistic regression models were constructed to identify factors associated with SDUI, stratified by self-reported HIV serostatus of the participants. The following variables were assessed in the bivariable models: concurrency (individual, perceived partner), sociodemographics, substance use, number of male sex partners, sex partner type, and frequency of sex with partner in last 3 months. Characteristics that were significant with p-value $₫ 0.10$ in the bivariable models were included in the multivariable models. A backward elimination process, with retention of variables at $\mathrm{p} \$ \mathbf{\$ 0} .05$, was used to generate the final multivariable models. Collinearity between variables was assessed using the variance inflation factors. All analyses were conducted in SAS version 9.3 (SAS Institute Inc., Cary, NC, USA).

\section{RESULTS}

Between 2010-2013, 4,998 men provided contact information; 1,997 met the study's eligibility criteria and scheduled a study visit. 1,503 men (75.3\%) enrolled. After excluding men who did not complete the SSNQ and who did not report any sex partners in the last 3 months, 1,458 (97.0\%) were included in this analysis.

\section{Baseline Individual, Partner, and Risk Behavior Characteristics}

Median age was 29 years (Table 1). White, non-Hispanic men comprised 32.1\%; Hispanic $30.1 \%$; Black, non-Hispanic $25.1 \%$; other race/ethnicity $12.7 \% .23 .5 \%$ reported being HIVpositive. Among the 1,077 men who agreed to HIV testing, 23 (2.1\%) were newly diagnosed with HIV (i.e., self-reported HIV negative on the ACASI questionnaire). The men reported mean 3.2 male sex partners in last 3 months. Very few reported having female or transgender partners. 22.6\% reported having an HIV-positive partner in the last 3 months. $39.4 \%$ reported having a main partner, while $30.3 \%$ reported having an anonymous sex partner. Over $60 \%$ reported meeting a sex partner on the internet or mobile application. $22.4 \%$ reported engaging in any UAI with a male partner in the last 3 months; $16.6 \%$ reported having SDUI with a male, female, or transgender partner.

\section{Prevalence of Concurrency}

The proportion who reported individual concurrency based on the single question (59.6\%) and dates $(63.2 \%)$ was fairly similar; fewer men $(0.3 \%)$ reported "don't know" to the single 
question than men who had missing start and/or end dates (2.0\%) (Table 2). The kappa coefficient between the two measures of concurrency was substantial $(\kappa=0.70) .71 .5 \%$ reported perceived partner concurrency, with only $3.8 \%$ who reported "don't know" or had missing data. $56.1 \%$ reported that both they and their partners had concurrent partners (reciprocal concurrency); $7.1 \%$ reported having individual concurrency only; $15.4 \%$ reported having perceived partner concurrency only; $18.5 \%$ reported having neither individual nor perceived partner concurrency. Correlation between individual and perceived partner concurrency was not high $(r=0.52)$. The median number of concurrent partners was 3 for all forms of concurrency.

\section{Comparison of Concurrency by Sociodemographic Characteristics and HIV Serostatus}

Men who reported individual concurrency were more likely to have a higher education level than those who did not report individual concurrency; the same finding was observed with reciprocal concurrency (Table 3). Men who reported perceived partner concurrency were more likely to be older, to self-report being HIV-positive, and to be newly or previously HIV-positive on HIV testing compared with men who did not report perceived partner concurrency. No differences in prevalence of concurrency were found by race/ethnicity, sexual orientation, and household income.

\section{Correlates of SDUI}

Collinearity of variables in the models for SDUI was low, with variance of inflation factor for all variables <2. In multivariable analysis among HIV-positive men by self-report, the following variables were positively associated with having SDUI in the last 3 months (Table 4): having individual concurrency, any alcohol use during sex, having more male sex partners, and not having a main partner in the last 3 months. Among HIV-negative men by self-report, having SDUI in the last 3 months was positively associated with having perceived partner concurrency, having a lower education level, any alcohol use during sex, any drug use during sex, having more male sex partners, and having an anonymous partner in the last 3 months; younger men and those who reported marijuana use in the last 3 months were less likely to report having SDUI.

\section{DISCUSSION}

In this study, concurrent partnerships were common among MSM: $63.2 \%$ reported concurrent sex partners, $71.5 \%$ reported partners whom they believed had concurrent partners, and $56.1 \%$ reported both they and their partners had concurrent partners in the last 3 months. Although direct comparison is constrained by various definitions and measures of concurrency used in different studies, our findings are qualitatively comparable to or higher than those reported among other MSM. ${ }^{19,20,26}$ In the San Francisco study, 78\% of MSM reported that they were having sex with other people during the last 12 months while they were having a sexual relationship with their most recent partner and 64\% reported any concurrency using a partner-by-partner approach. ${ }^{19}$ An online study of MSM showed a 45\% prevalence of concurrent partnerships, based on data on up to 5 most recent partners in the last 6 months. ${ }^{20}$ In another study, prevalence of concurrency (defined as respondents who reported any overlapping partnerships based on dates in the last year) among MSM ranged 
from $18 \%$ to $31 \%$, much higher than concurrency prevalence among heterosexual men and women of $9.7 \%$ and $7.5 \%$, respectively. ${ }^{27}$ A similarly low prevalence (11\%) of concurrency in the past 12 months among heterosexual men in the U.S. was reported in another study. ${ }^{4}$ The men in our study not only reported high levels of individual, perceived partner, and reciprocal concurrency overall, but also reported relatively high numbers of concurrent partners among those who reported concurrency, with a median of 3 concurrent partners (a vast majority of whom were male) for each form of concurrency. The high prevalence of concurrency and large number of concurrent partners observed in our study might be explained by recruitment and enrollment of men with riskier sexual behaviors, owing to our study's eligibility criterion that the men engage in anal sex in the last 3 months, as opposed to the last 6 or 12 months used in other studies.

Our study finding that over half reported having reciprocal concurrency is unique. These men reported involvement in concurrent sexual relationships with partners whom they also believed to be in concurrent partnerships themselves. Not only are these men at possibly increased risk for HIV and other STIs by having concurrent sexual relationships, but they may also be at risk indirectly through their partner's concurrency. ${ }^{10}$ These data imply that many men are members of potentially extensive, interconnected sexual networks in which HIV and other STIs have the capability to disseminate efficiently, especially if they go undiagnosed, untreated, or inadequately treated and if they practice versatility in anal sex roles, and in which a large number of people can be affected. ${ }^{5,27,28}$ Moreover, there may be the potential for "sexual bridging" by linking networks with dissimilar levels of risk behaviors and prevalence of HIV and other STIs, thus facilitating the movement of HIV and STI epidemics from those at higher risk to those at lower risk. ${ }^{5}$

Key HIV risk factors, including alcohol and drug use during sex, having an anonymous partner, and having a greater number of male sex partners, ${ }^{18,29}$ were positively associated with SDUI. We found individual concurrency to be positively associated with SDUI among self-reported HIV-positive men and perceived partner concurrency to be positively associated with SDUI among self-reported HIV-negative men. The association of individual and perceived partner concurrency with SDUI suggests that concurrency can lead to increased HIV acquisition and transmission risk within, and across, populations. Our results are similar to several studies in which an association between concurrency and UAI in MSM was observed. ${ }^{10,19,30}$

In our study, we did not find any difference in prevalence of concurrency by race/ethnicity. In the U.S., the disproportionate rate of HIV infection among black MSM is not explained solely by differences in individual risk behaviors. Millett et al had posited that the sexual networks (of which concurrency is a structural component) of black MSM may place them at increased risk of HIV infection compared to non-black MSM. ${ }^{31,32}$ Similar to our study, the online study among MSM found no differences by race/ethnicity in the 6-month period prevalence of concurrency and concurrent UAI. ${ }^{20}$ Interestingly, in another analysis using the same online study sample and incorporating measurements of indirect exposure of the men's partners to other partners, the authors found that partners of black MSM, compared to partners of White MSM, were more likely to be linked to other partners through UAI and as a result of concurrency. ${ }^{10}$ In another study, black men had fewer sexual partners but were 3 
times more likely to report complete concurrency (defined as complete overlap within 3 weeks of the last 5 partnerships) compared with non-black MSM. ${ }^{19}$

We observed a high level of agreement between our two measures of individual concurrency: one based on the single, direct question and the other based on overlapping dates. Definitions, measures, and indicators of concurrency varied in previous concurrency studies, and it remains unclear which correlate best with HIV risk. ${ }^{1}$ The UNAIDS recommended measurement of concurrent partnerships using a point prevalence 6 months before interview assessment (rather than current concurrency) and based on overlapping dates. The UNAIDS also proposed that data could be limited to the 3 most recent partners. These recommendations, however, have potential measurement bias. ${ }^{3,33}$ In a Malawi population-based survey study of different measurements of concurrency, ${ }^{3}$ the concurrency measure based on overlapping dates yielded lower concurrency estimates that those that queried directly about concurrency partnerships for each relationship. This underestimation was attributed to incomplete reporting of all partners and difficulty in recalling dates. It is unclear whether and how the UNAIDS recommendations for concurrency measurement apply directly to concurrency research among MSM.

Our study has several limitations. Participants might not be representative of all MSM living in NYC. Use of a systematic sampling scheme in our study should minimize this selection bias. Self-reported data on the ACASI and data reported to interviewers on the SSNQ might not accurately reflect the actual risk behaviors of the participant and of their partners. The ACASI system but not the SSNQ would tend to reduce socially desirable responding. ${ }^{34,35}$ It is possible that report of partners' information was inaccurate, especially related to perceived partner concurrency, by participants without mechanisms to verify the information directly from the partners. Past studies have employed different measures of concurrency, so direct comparisons of these findings with past studies in heterosexual and MSM populations are restricted. Another limitation is the lack of a biological outcome, such as incident HIV and STI infections, to relate to concurrency. Our logistic regression models provided correlates of SDUI (which included concurrency) without inferences about causality. Finally, our analysis was restricted to anal and vaginal sex and excluded oral sex; we also excluded examination of antiretroviral use among HIV-positive men and pre-exposure prophylaxis use among HIV-negative men. These exclusions might underestimate concurrency prevalence in individuals who use oral sex or antiretroviral medications as risk reduction strategies.

\section{CONCLUSION}

We found a high prevalence of concurrent partnerships in the last 3 months among MSM in NYC. We observed individual and perceived partner concurrency as well as other factors, such as alcohol and drug use during sex, having an anonymous partner, and having a greater number of sex partners, to be significantly associated with SDUI. Our results provide additional insights into concurrency among MSM in the U.S., and highlight the importance of research to further understand the risk of HIV acquisition and transmission conferred by concurrency. ${ }^{20} \mathrm{HIV}$ prevention interventions that directly address concurrency among MSM in general as well as specific high-risk MSM subpopulations should be considered. 


\section{Acknowledgments}

This study was supported by NIH K01 DA031035 (HVT) and R01 HD059729.

\section{References}

1. UNAIDS Reference Group on Estimates MaP. Consultation on concurrent partnerships. 2009

2. Unaids Reference Group on Estimates M. Projections: Working Group on Measuring Concurrent Sexual P. HIV: consensus indicators are needed for concurrency. Lancet. 2010; 375:621-622. [PubMed: 19954832]

3. Glynn JR, Dube A, Kayuni N, et al. Measuring concurrency: an empirical study of different methods in a large population-based survey and evaluation of the UNAIDS guidelines. Aids. 2012; 26:977-985. [PubMed: 22555149]

4. Aral SO. Partner Concurrency and the STD/HIV Epidemic. Current infectious disease reports. 2010; 12:134-139. [PubMed: 21308509]

5. Gorbach PM, Holmes KK. Transmission of STIs/HIV at the partnership level: beyond individuallevel analyses. J Urban Health. 2003; 80:iii15-iii25. [PubMed: 14713668]

6. Doherty IA, Padian NS, Marlow C, Aral SO. Determinants and consequences of sexual networks as they affect the spread of sexually transmitted infections. The Journal of infectious diseases. 2005; 191(Suppl 1):S42-S54. [PubMed: 15627230]

7. Manhart LE, Aral SO, Holmes KK, Foxman B. Sex partner concurrency: measurement, prevalence, and correlates among urban 18-39-year-olds. Sexually transmitted diseases. 2002; 29:133-143. [PubMed: 11875374]

8. Morris M, Kurth AE, Hamilton DT, Moody J, Wakefield S. Concurrent partnerships and HIV prevalence disparities by race: linking science and public health practice. American journal of public health. 2009; 99:1023-1031. [PubMed: 19372508]

9. Morris M, Kretzschmar M. Concurrent partnerships and the spread of HIV. Aids. 1997; 11:641-648. [PubMed: 9108946]

10. Rosenberg ES, Rothenberg RB, Kleinbaum DG, Stephenson RB, Sullivan PS. The implications of respondent concurrency on sex partner risk in a national, web-based study of men who have sex with men in the United States. Journal of acquired immune deficiency syndromes. 2013; 63:514521. [PubMed: 23591633]

11. Goodreau SM, Cassels S, Kasprzyk D, Montano DE, Greek A, Morris M. Concurrent partnerships, acute infection and HIV epidemic dynamics among young adults in Zimbabwe. AIDS and behavior. 2012; 16:312-322. [PubMed: 21190074]

12. Mah TL, Halperin DT. Concurrent sexual partnerships and the HIV epidemics in Africa: evidence to move forward. AIDS and behavior. 2010; 14:11-16. dicussion 34-7. [PubMed: 18648926]

13. Kenyon C, Buyze J, Colebunders R. HIV Prevalence by Race Co-Varies Closely with Concurrency and Number of Sex Partners in South Africa. PLoS One. 2013; 8:e64080. [PubMed: 23704973]

14. Sawers L, Stillwaggon E. Concurrent sexual partnerships do not explain the HIV epidemics in Africa: a systematic review of the evidence. Journal of the International AIDS Society. 2010; 13:34. [PubMed: 20836882]

15. Lurie MN, Rosenthal S. Concurrent partnerships as a driver of the HIV Epidemic in sub-Saharan Africa? The evidence is limited. AIDS and behavior. 2010; 14:17-24. discussion 5-8. [PubMed: 19488848]

16. Lagarde E, Auvert B, Carael M, et al. Concurrent sexual partnerships and HIV prevalence in five urban communities of sub-Saharan Africa. Aids. 2001; 15:877-884. [PubMed: 11399960]

17. Tanser F, Barnighausen T, Hund L, Garnett GP, McGrath N, Newell ML. Effect of concurrent sexual partnerships on rate of new HIV infections in a high-prevalence, rural South African population: a cohort study. Lancet. 2011; 378:247-255. [PubMed: 21763937]

18. Koblin BA, Husnik MJ, Colfax G, et al. Risk factors for HIV infection among men who have sex with men. Aids. 2006; 20:731-739. [PubMed: 16514304] 
19. Bohl DD, Raymond HF, Arnold M, McFarland W. Concurrent sexual partnerships and racial disparities in HIV infection among men who have sex with men. Sex Transm Infect. 2009; 85:367-369. [PubMed: 19773457]

20. Rosenberg ES, Khosropour CM, Sullivan PS. High prevalence of sexual concurrency and concurrent unprotected anal intercourse across racial/ethnic groups among a national, Web-based study of men who have sex with men in the United States. Sexually transmitted diseases. 2012; 39:741-746. [PubMed: 23001260]

21. Maulsby C, Sifakis F, German D, Flynn CP, Holtgrave D. Partner characteristics and undiagnosed HIV seropositivity among men who have sex with men only (MSMO) and men who have sex with men and women (MSMW) in Baltimore. AIDS and behavior. 2012; 16:543-553. [PubMed: 21964976]

22. Whittington, WLH.; Sanchez, J.; Davis, A., et al. High prevalence of HSV-2 infection and high rates of partner concurrency among Peruvian men who have sex with men (MSM). 5th Biennial Congress, International Society for Sexually Transmitted Diseases Research; Ottawa, Canada. 2003. Abstract No. 0693.

23. Choi KH, Ning Z, Gregorich SE, Pan QC. The influence of social and sexual networks in the spread of HIV and syphilis among men who have sex with men in Shanghai, China. Journal of acquired immune deficiency syndromes. 2007; 45:77-84. [PubMed: 17325608]

24. Koblin BA, Egan JE, Rundle A, et al. Methods to measure the impact of home, social, and sexual neighborhoods of urban gay, bisexual, and other men who have sex with men. PLoS One. 2013; 8:e75878. [PubMed: 24146785]

25. MacKellar DA, Gallagher KM, Finlayson T, Sanchez T, Lansky A, Sullivan PS. Surveillance of HIV risk and prevention behaviors of men who have sex with men--a national application of venue-based, time-space sampling. Public Health Rep. 2007; 122(Suppl 1):39-47. [PubMed: 17354526]

26. Gorbach PM, Murphy R, Weiss RE, Hucks-Ortiz C, Shoptaw S. Bridging sexual boundaries: men who have sex with men and women in a street-based sample in Los Angeles. J Urban Health. 2009; 86(Suppl 1):63-76. [PubMed: 19543837]

27. Glick SN, Morris M, Foxman B, et al. A comparison of sexual behavior patterns among men who have sex with men and heterosexual men and women. Journal of acquired immune deficiency syndromes. 2012; 60:83-90. [PubMed: 22522237]

28. Grieb SM, Davey-Rothwell M, Latkin CA. Social and sexual network characteristics and concurrent sexual partnerships among urban African American high-risk women with main sex partners. AIDS and behavior. 2012; 16:882-889. [PubMed: 21861193]

29. Morris SR, Little SJ. MSM: resurgent epidemics. Current opinion in HIV and AIDS. 2011; 6:326332. [PubMed: 21537172]

30. Choi KH, Hudes ES, Steward WT. Social discrimination, concurrent sexual partnerships, and HIV risk among men who have sex with men in Shanghai, China. AIDS and behavior. 2008; 12:S71S77. [PubMed: 18427972]

31. Millett GA, Flores SA, Peterson JL, Bakeman R. Explaining disparities in HIV infection among black and white men who have sex with men: a meta-analysis of HIV risk behaviors. AIDS. 2007; 21:2083-2091. [PubMed: 17885299]

32. Millett GA, Peterson JL, Wolitski RJ, Stall R. Greater risk for HIV infection of black men who have sex with men: a critical literature review. Am J Public Health. 2006; 96:1007-1019. [PubMed: 16670223]

33. Morris M, Epstein H, Wawer M. Timing is everything: international variations in historical sexual partnership concurrency and HIV prevalence. PLoS One. 2010; 5:e14092. [PubMed: 21124829]

34. Ghanem KG, Hutton HE, Zenilman JM, Zimba R, Erbelding EJ. Audio computer assisted self interview and face to face interview modes in assessing response bias among STD clinic patients. Sexually transmitted infections. 2005; 81:421-425. [PubMed: 16199744]

35. Metzger DS, Koblin B, Turner C, et al. Randomized controlled trial of audio computer-assisted self-interviewing: utility and acceptability in longitudinal studies. HIVNET Vaccine Preparedness Study Protocol Team. American journal of epidemiology. 2000; 152:99-106. [PubMed: 10909945] 


\section{Table 1}

Baseline Sociodemographic, HIV Serostatus, and Risk Behavior Characteristics among MSM, NYC M2M Study $(\mathrm{N}=1458)$

\begin{tabular}{|c|c|}
\hline & $\mathbf{N}(\%)$ \\
\hline \multicolumn{2}{|l|}{ From ACASI Questionnaire: } \\
\hline $\begin{array}{l}\text { Age (years) } \\
18 \text { to } 24 \\
25 \text { to } 29 \\
30 \text { to } 39 \\
40+\end{array}$ & $\begin{array}{l}379(26.0) \\
397(27.3) \\
347(23.8) \\
334(22.9)\end{array}$ \\
\hline $\begin{array}{l}\text { Race/ethnicity } \\
\text { White, non-Hispanic } \\
\text { Hispanic } \\
\text { Black, non-Hispanic } \\
\text { Other/mixed race }\end{array}$ & $\begin{array}{l}467(32.1) \\
437(30.1) \\
365(25.1) \\
184(12.7)\end{array}$ \\
\hline $\begin{array}{l}\text { Sexual Orientation } \\
\text { Queer/homosexual } \\
\text { Bisexual } \\
\text { Not sure/other/don't know }\end{array}$ & $\begin{array}{c}1277(87.6) \\
145(10.0) \\
36(2.5)\end{array}$ \\
\hline $\begin{array}{l}\text { Annual household Income (USD) } \\
<\$ 10,000 \\
\$ 10,000-<\$ 40,000 \\
\$ 40,000-<\$ 60,000 \\
\geq \$ 60,000\end{array}$ & $\begin{array}{l}275(19.8) \\
519(37.4) \\
368(26.5) \\
227(16.3)\end{array}$ \\
\hline $\begin{array}{l}\text { Education } \\
\text { Less than high school graduate } \\
\text { High school graduate } \\
\text { Some college } \\
\text { College graduate or more }\end{array}$ & $\begin{array}{c}80(5.5) \\
162(11.1) \\
490(33.6) \\
726(49.8)\end{array}$ \\
\hline $\begin{array}{l}\text { Self-reported HIV status } \\
\text { Positive } \\
\text { Negative }\end{array}$ & $\begin{array}{c}327(23.5) \\
1063(76.5)\end{array}$ \\
\hline $\begin{array}{l}\text { HIV status by HIV testing } a(\mathrm{~N}=1077) \\
\text { Newly HIV positive } \\
\text { HIV negative } \\
\text { Previously HIV positive }\end{array}$ & $\begin{array}{c}23(2.1) \\
855(79.4) \\
199(18.5)\end{array}$ \\
\hline Any sexually transmitted infection ${ }^{b}$ in last 3 months & $118(8.1)$ \\
\hline $\begin{array}{l}\text { Any alcohol and drug use in the last } 3 \text { months } \\
\text { Alcohol } \\
\text { Marijuana } \\
\text { Inhaled nitrites, poppers } \\
\text { Powder cocaine, coke } \\
\text { Vicodin, Oxycontin, Xanax, or other opiates or benzodiazepines } \\
\text { Club drugs } \\
\text { Any injection drug use } \\
\text { Methamphetamine } \\
\text { Smoked cocaine, crack, freebase } \\
\text { Heroin }\end{array}$ & $\begin{array}{l}1319(90.6) \\
760(52.2) \\
500(34.3) \\
283(19.4) \\
185(12.7) \\
161(11.0) \\
9(0.6) \\
78(5.4) \\
39(2.7) \\
2(0.1)\end{array}$ \\
\hline \multicolumn{2}{|l|}{ From SSNQ: } \\
\hline Any alcohol use during unprotected sex with a partner in last 3 months & $371(25.5)$ \\
\hline Any drug use during unprotected sex with a partner in last 3 months & $310(21.3)$ \\
\hline Total number of male sexual partners in last 3 months, mean (SD) ${ }^{c}$ & $3.2(2.8)$ \\
\hline More than 15 sex partners in last 3 months & $21(1.4)$ \\
\hline Any female sex partners in last 3 months & $35(2.4)$ \\
\hline Any transgender partners in last 3 months & $15(1.0)$ \\
\hline
\end{tabular}




\begin{tabular}{|l|c|}
\hline & N (\%) \\
\hline Any HIV+ sex partners in last 3 months & $330(22.6)$ \\
\hline Any exchange or trade partners $d$ in last 3 months & $47(3.2)$ \\
\hline Any anonymous partners $e$ in last 3 months & $442(30.3)$ \\
\hline Any main partners $f$ in last 3 months & $575(39.4)$ \\
\hline Any sex partners met on the internet or mobile application in last 3 months & $887(60.8)$ \\
\hline Any serodiscordant/serostatus unknown sexual relationships in last 3 months & $633(43.4)$ \\
\hline Any $\mathrm{UAI}^{g}$ in last 3 months & $326(22.4)$ \\
\hline Any SDUI ${ }^{h}$ in last 3 months & $242(16.6)$ \\
\hline Any frequent ${ }^{i}$ anal or vaginal sex with at least one partner in last 3 months & $224(15.4)$ \\
\hline
\end{tabular}

${ }^{a}$ HIV status by HIV testing among those men who agreed to HIV testing. A total of 381 men refused HIV testing: 131 (34.4\%) were men who selfreported being HIV positive, while $225(59.1 \%)$ were men who self-reported being HIV negative; the remainder reported never testing for HIV in the past or reported having indeterminate results.

${ }^{b}$ Self-report history of syphilis, genital or rectal gonorrhea or chlamydia, new genital or rectal herpes in last 3 months

${ }^{c}$ Mean (SD) for total number of sexual partners (male, female, transgender) in last 3 months, 3.3 (2.8)

$d_{\text {Exchange or trade partner was defined as "a person you had anal or vaginal sex with in exchange for something like cash, drugs, a meal or a place }}$ to stay" on the SSNQ

${ }^{e}$ An anonymous partner was defined as “a person you hooked up with just to have anal or vaginal sex and you don't know or don't remember their first and/or last name" on the SSNQ.

${ }^{f}$ Main partner was defined as "a person that you have lived with or have seen a lot, whom you have had anal or vaginal sex with, and to whom you have felt a special emotional commitment" on the SSNQ.

${ }^{g}$ UAI: any unprotected receptive or insertive anal sex in last 3 months

$h_{\text {SDUI: serodiscordant/serostatus unknown unprotected anal and/or vaginal intercourse }}$

${ }^{i}$ A participant was categorized as having frequent anal or vaginal sex if he answered daily, several times per week, or weekly in the last 3 months with at least one partner to the question, "How often did you (have anal or vaginal sex, top [your penis in his anus], bottom [his penis in your anus]) with [name] in the past 3 months? With and without a condom and with or without ejaculation."

Columns may not add up to total due to missing data. 
Table 2

Prevalence of Individual, Perceived Partner, and Reciprocal Concurrency, NYC M2M Study (N=1458)

\begin{tabular}{|l|c|}
\hline & $\mathbf{N}(\boldsymbol{\%})$ \\
\hline Individual concurrency $^{a}$ & $869(59.6)$ \\
Based on single question: & $585(40.1)$ \\
Yes & $4(0.3)$ \\
No & $921(63.2)$ \\
Don't know/missing & $508(34.8)$ \\
Based on dates: & $29(2.0)$ \\
Yes & \\
No & \\
Don't know/missing & \\
\hline Perceived partner concurrency & $1043(71.5)$ \\
Yes & $360(24.7)$ \\
No & $55(3.8)$ \\
Don't know/missing & \\
\hline Reciprocal concurrency & $818(56.1)$ \\
Yes (individual and perceived partner concurrency) & $103(7.1)$ \\
No & $225(15.4)$ \\
Individual concurrency only (no perceived partner concurrency) & $270(18.5)$ \\
Perceived partner concurrency only (no individual concurrency) & $42(2.9)$ \\
No individual and no perceived partner concurrency & \\
Don't know/missing & \\
\hline Number of concurrent partners (among those with concurrency) & $5.0(6.2)$ \\
Among those with individual concurrency based on single question: & $3(2-5)$ \\
Mean (SD) number of concurrent partners & $4.5(6.0)$ \\
Median (IQR) & $3(2-5)$ \\
Among those with individual concurrency based on dates: & $4.3(5.9)$ \\
Mean (SD) & $3(2-5)$ \\
Median (IQR) & $4.9(6.2)$ \\
Among those with perceived partner concurrency: & $3(2-5)$ \\
Mean (SD) & \\
Median (IQR) & \\
Among those with reciprocal concurrency: & \\
Mean (SD) & \\
Median (IQR) & \\
\hline
\end{tabular}

${ }^{a}$ Kappa coefficient of agreement between the two measures of individual concurrency was 0.70 (95\%CI $0.66-0.74$ ).

Columns may not add up to total due to missing data.

NA: not applicable 


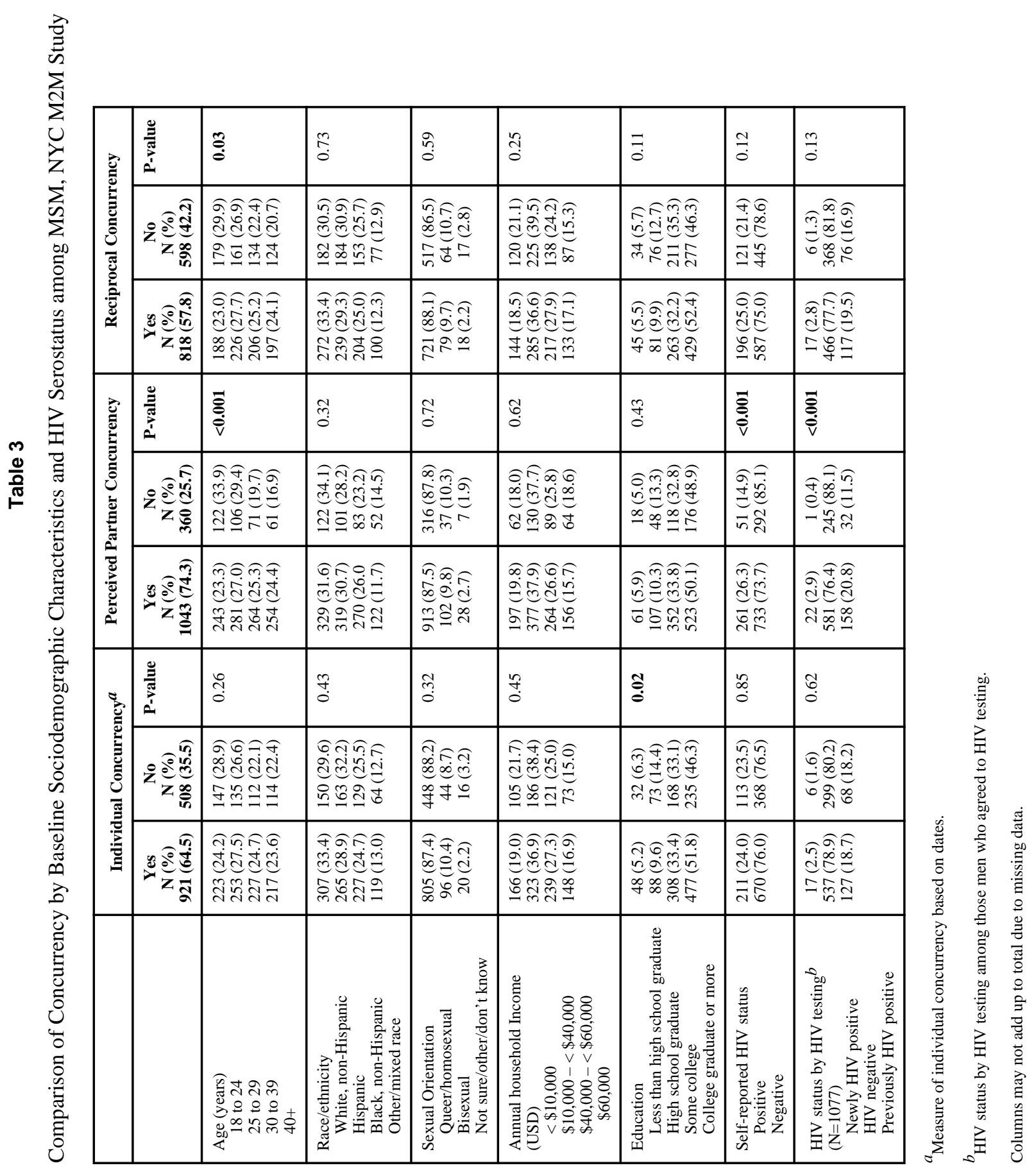




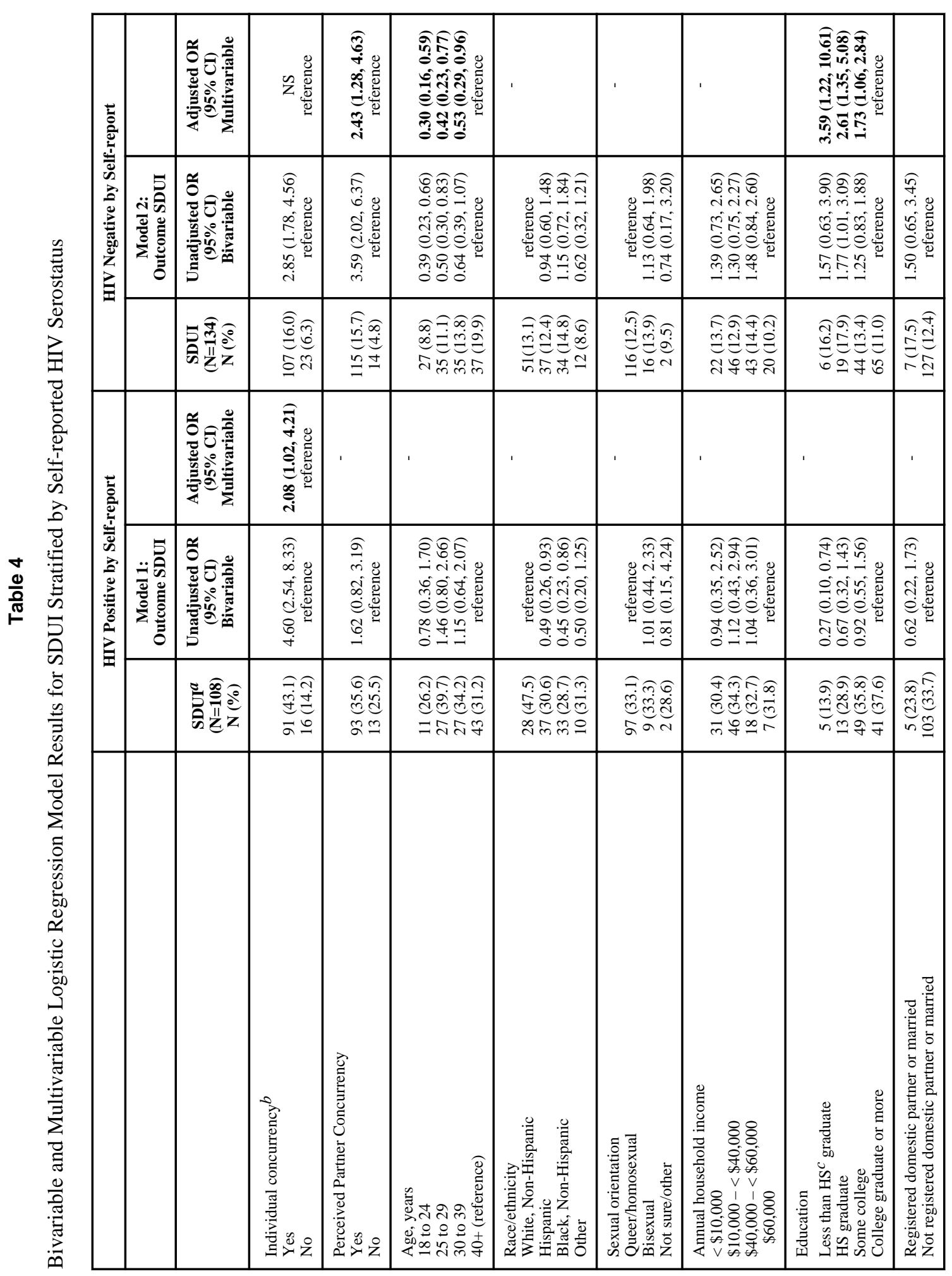




\begin{tabular}{|c|c|c|c|c|c|c|c|c|c|c|c|}
\hline \multirow{3}{*}{ 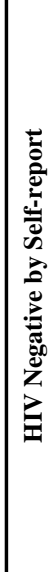 } & & 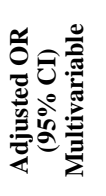 & 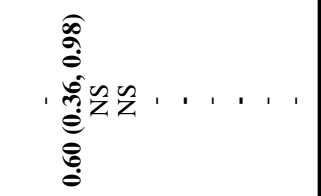 & 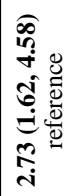 & 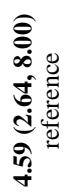 & 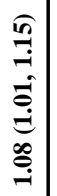 & & 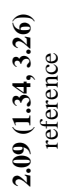 & & 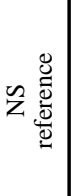 & \\
\hline & 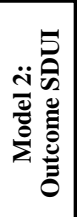 & 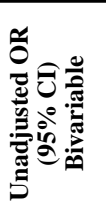 & 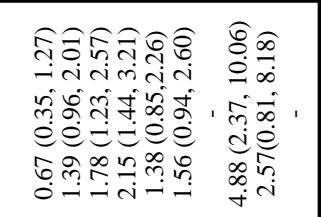 & 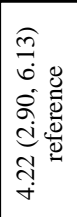 & 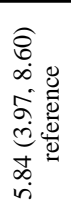 & 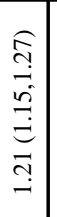 & 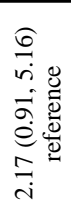 & 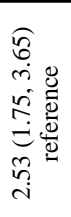 & 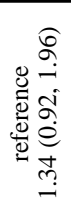 & 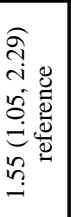 & 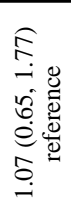 \\
\hline & & 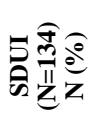 & 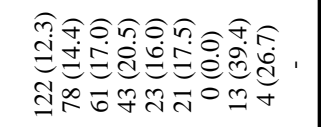 & 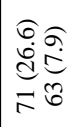 & 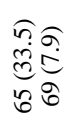 & $\begin{array}{l}\sigma \\
\stackrel{0}{0} \\
\stackrel{+}{+}\end{array}$ & 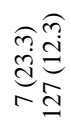 & 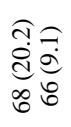 & 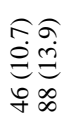 & 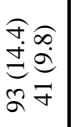 & 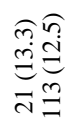 \\
\hline \multirow{4}{*}{ 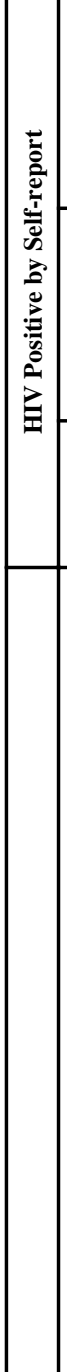 } & & 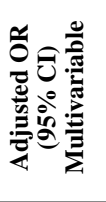 & & 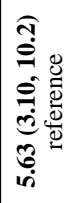 & 纪总 & 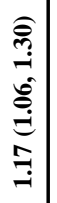 & & 纪总 & 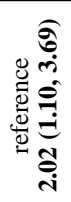 & 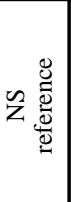 & \\
\hline & 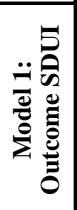 & 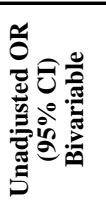 & 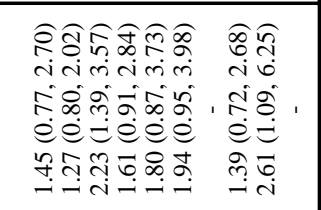 & 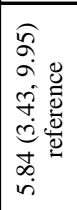 & 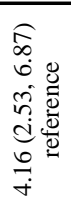 & 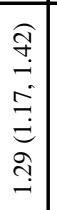 & 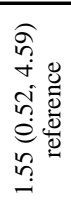 & 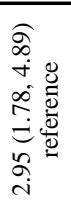 & 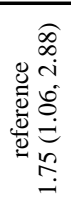 & 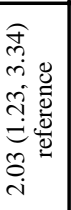 & 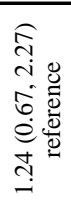 \\
\hline & & 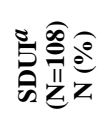 & 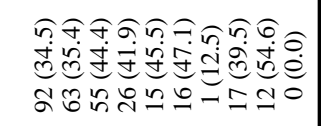 & $\begin{array}{l}\text { of } \\
\text { ded } \\
\begin{array}{c}d \\
\text { ind }\end{array}\end{array}$ & 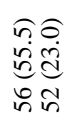 & $\begin{array}{c}\overparen{E} \\
\stackrel{0}{0} \\
\dot{+} \\
\dot{+}\end{array}$ & 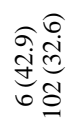 & 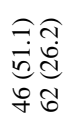 & 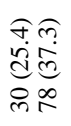 & 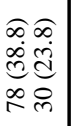 & 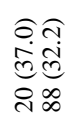 \\
\hline & & & 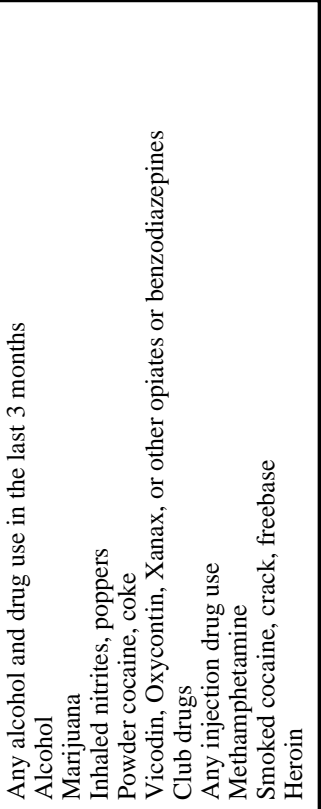 & 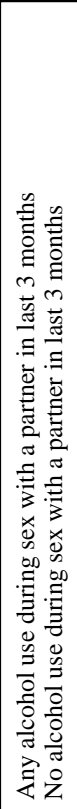 & 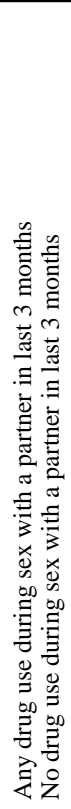 & 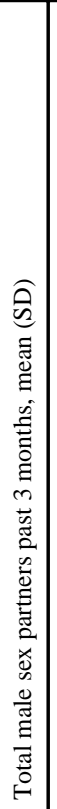 & 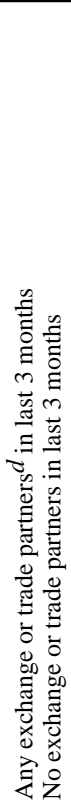 & 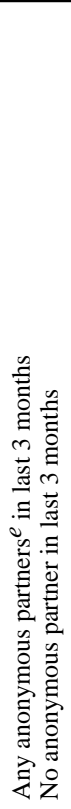 & 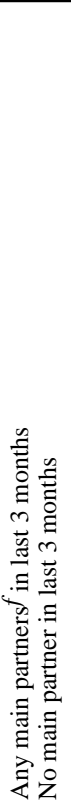 & 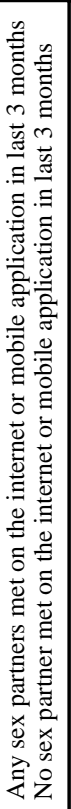 & 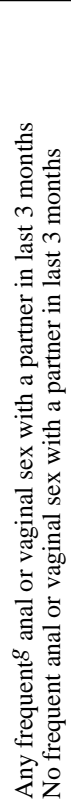 \\
\hline
\end{tabular}

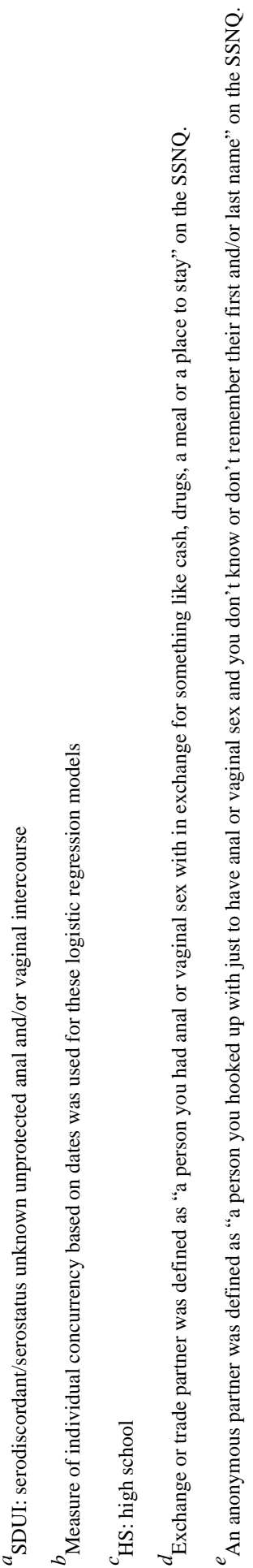




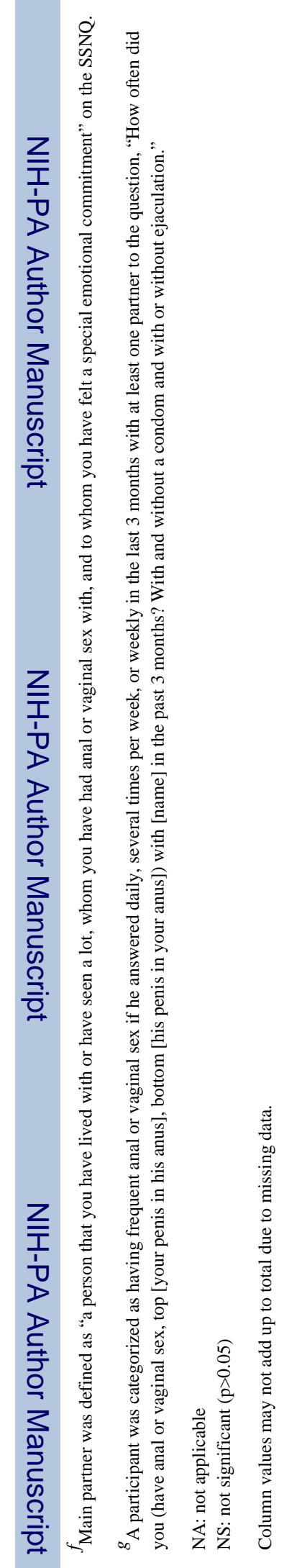

